\title{
11.4 \\ Измерение мощности сверхширокополосного хаотического сигнала для решения задач определения расстояния и позиционирования
}

\author{
(C) Е.В. Ефрремова, Л.В. Кузьмин \\ Институт радиотехники и электроники им. В.А. Котельникова РАН, Москва, Россия \\ E-mail: efremova@cplire.ru
}

Поступило в Редакцию 21 августа 2020г.

В окончательной редакции 20 февраля 2021 г.

Принято к публикации 20 фревраля 2021 г.

\begin{abstract}
Предложена методика увеличения точности измерения расстояния при помощи сверхширокополосных хаотических радиоимпульсов, предназначенная для применения в беспроводных сенсорных сетях. В результате проведенных экспериментальных стендовых исследований показано, что уровень относительной ошибки измерения расстояния находится в пределах 5-9\%.
\end{abstract}

Ключевые слова: сверхширокополосные сигналы, беспроводной канал, сенсорные сети, измерение расстояния, сверхширокополосная несущая, хаотические сверхширокополосные сигналы.

DOI: 10.21883/PJTF.2021.10.50970.18524

Актуальной проблемой в современных беспроводных сетях малого радиуса действия (локальные беспроводные сети) является определение расстояния между узлами сети [1]. В зависимости от назначения системы и диапазона измеряемых расстояний могут применяться радиосигналы различных типов и частотных диапазонов (технологии Bluetooth, Wi-Fi, RFID, UWD [2]). Выбор в пользу того или иного метода измерения осуществляется на основе компромисса между требуемой точностью, дальностью и сложностью реализации. В частности, привлекательными являются решения, основанные на измерении мощности радиосигнала, благодаря их относительной физико-технической простоте. Поэтому они лежат в основе различных методов позиционирования объектов в беспроводных сетях, когда параметры канала известны (fingerprinting) [3-5], неизвестны [6,7] или неизвестна мощность излучаемого сигнала [8].

Принципиальными задачами, находящимися в фокусе внимания исследователей, являются повышение точности измерения мощности радиосигнала и расширение диапазона по расстоянию. Их успешному решению препятствуют негативное влияние многолучевого распространения и явление замирания радиосигналов сверхвысокочастотного диапазона, причем повышение точности измерения мощности сигнала (и соответствующего ему расстояния) в режиме точка-точка на несколько процентов позволяет многократно увеличить точность определения координат объекта относительно множества (кластера) узлов в беспроводной сети $[9,10]$.

В настоящее время технологии беспроводного позиционирования позволяют обеспечить следующую медианную [11] точность: Wi-Fi $<2 \mathrm{~m}$; методы, использующие fingerprinting, $<5 \mathrm{~m}$; Bluetooth $-2-5 \mathrm{~m}$; технологии RFID $<2 \mathrm{~m}$; беспроводные сенсорные сети, работающие в кооперативном режиме, $<2 \mathrm{~m}$; ZigBee $<5 \mathrm{~m}$; UWB-устройства $<50 \mathrm{~cm}$.
Одно из направлений увеличения точности - расширение спектра сигнала вплоть до сверхширокополосного (СШП) диапазона, компенсирующего последствия многолучевой интерференции и снижающего флуктуации мощности сигнала в точке приема. Хаотический (шумоподобный) сигнал [12] в этом смысле является практически идеальным кандидатом для достижения данной цели. В [13] описан метод определения расстояния между СШП-приемопередатчиками, основанный на измерении мощности хаотического СШП-сигнала.

Целью данного исследования является разработка методики увеличения точности измерения расстояния по мощности и расширения диапазона работы метода [13] по расстоянию до величин, которые интересны для практических приложений.

Определение расстояния $d$ при помощи измерения мощности $P$ сигнала основано на зависимости падения мощности сигнала от расстояния

$$
d=d_{0} \cdot 10^{\left(P_{d}-P_{0}\right) /(10 n)}=d_{0} \cdot 10^{\left(A_{d}-A_{0}\right) /(10 n h)},
$$

где $P_{d}[\mathrm{dBm}]$ - мощность сигнала на расстоянии $d[\mathrm{~m}]$ от источника излучения (между передатчиком и приемником); $P_{0}[\mathrm{dBm}]-$ мощность сигнала на расстоянии $d_{0}[\mathrm{~m}] ; P_{0}$ и $d_{0}$ определяются в ходе измерений и являются референсными значениями, относительно которых производятся дальнейшие вычисления; $A_{d}[\mathrm{~V}]-$ амплитуда огибающей радиоимпульсов, имеющих мощность $P_{d} ; A_{0}[\mathrm{~V}]$ - амплитуда огибающей радиоимпульсов, имеющих мощность $P_{0} ; n-$ показатель затухания сигнала в канале связи; $h[\mathrm{~V} / \mathrm{dBm}]-$ крутизна характеристики энергетического детектора, осуществляющего преобразование мгновенной входной мощности в мгновенное напряжение [13].

Измеряемое приемником мгновенное напряжение является случайной величиной, которая определяется 


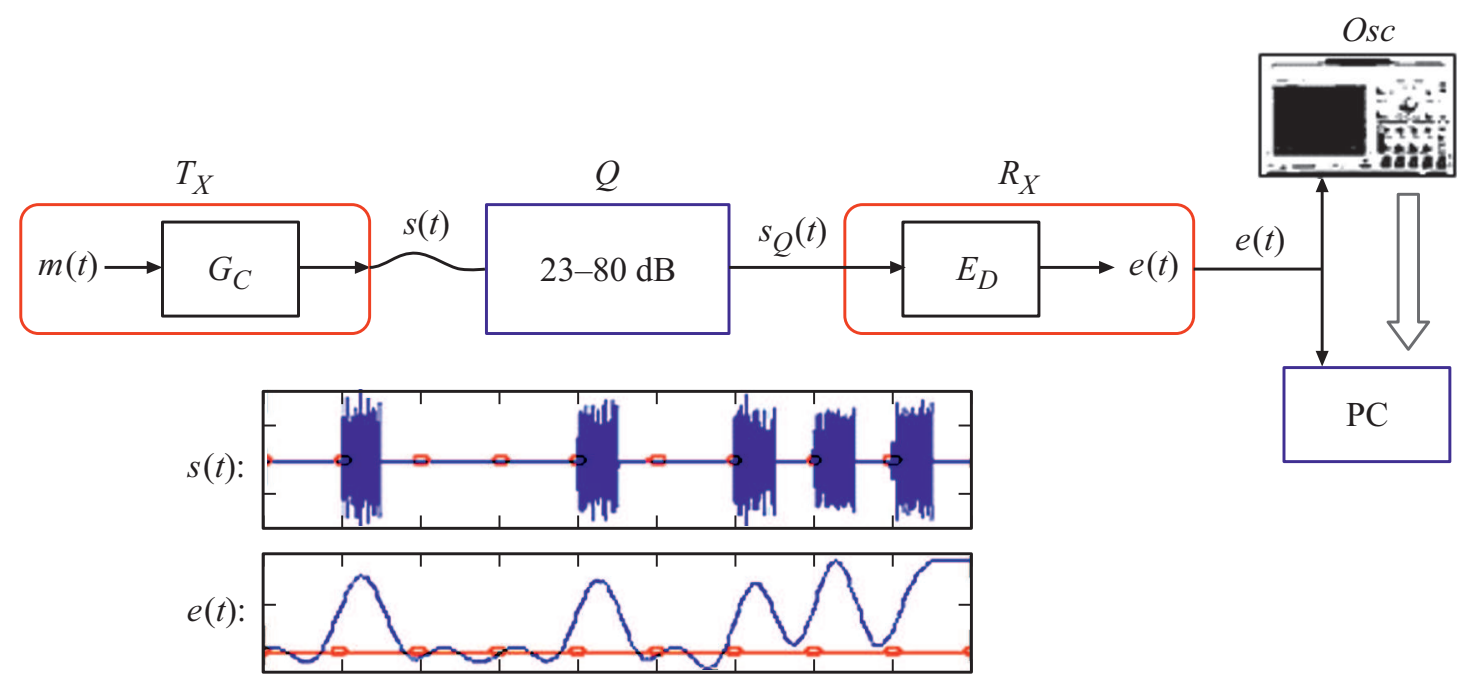

Рис. 1. Схема экспериментального макета. Пояснение в тексте.

мгновенным значением хаотического сигнала, воздействием канала и входных цепей приемника. На основе знания мгновенного значения огибающей сигнала в приемнике необходимо получить оценку амплитуды $A_{d}$ и мощности $P_{d}$ принятого сигнала.

В рамках излагаемого подхода используется косвенное измерение мощности $P_{d}$ через подсчет числа импульсов, которые имеют мощность не меньше заданной [13]: приемное устройство детектирует поступающие радиоимпульсы путем сравнения уровня принятого сигнала с некоторым пороговым значением $V_{T}$, подсчитывает число $p_{r}$ обнаруженных и число $p_{l}$ пропущенных импульсов (предполагается, что передаваемая последовательность импульсов известна) и вычисляет значение числа ошибок обнаружения

$$
F=p_{r} /\left(p_{r}+p_{l}\right) \text {. }
$$

Путем варьирования значения $V_{T}$ определяется значение $V_{T}^{*}$, при котором достигается заданное значение $F^{*}$. Полученное значение $V_{T}^{*}$ связано с уровнем мощности (амплитуды) радиоимпульсов.

Для того чтобы определить возможную достижимую точность измерения расстояния для такого способа оценки мощности сигнала, был проведен эксперимент, в котором изменялась мощность принимаемого сигнала и анализировались различные правила оценки амплитуды принятого сигнала, которые дали бы наиболее точный результат определения расстояния.

Экспериментальный макет (рис. 1) состоял из двух прямохаотических приемопередающих модулей [14], коаксиального кабеля длиной $1 \mathrm{~m}$, перестраиваемого аттенюатора, который имитировал ослабление сигнала в беспроводном канале, осциллографа $(O s c)$ и персонального компьютера (РС).

Передающее устройство $\left(T_{X}\right)$ формировало последовательность $s(t)$, состоящую из хаотических радиоимпульсов длительностью $T_{P}=166 \mathrm{~ns}$, мощностью $P_{P}=50 \mathrm{~mW}$ и периодом следования $2 T_{P}$. Сигнал передатчика по кабелю подавался на вход аттенюатора, вносящего ослабление $Q$, на выходе которого формировался сигнал $s_{Q}(t)$, который поступал на вход приемного устройства $\left(R_{X}\right)$. На выходе приемника формировалась огибающая $e(t)$ сигнала $s_{Q}(t)$.

Измерения проводились для набора ослаблений: $Q_{i}=\{23,29,35,41,47,53,59,65,71,74,77,80\}$ dВ. Указанный ряд значений выбран из соображений удобства анализа результатов: ослабление в $41 \mathrm{~dB}$ соответствует затуханию сигнала на частоте $4 \mathrm{GHz}$ на $1 \mathrm{~m}$. Остальные значения кратны изменению расстояния в 2 или в $\sqrt{2}$ раз при условии, что показатель затухания сигнала в канале $n=2$. Таким образом, указанным значениям затухания соответствуют эквивалентные расстояния $d_{i}$ от 0.125 до $89 \mathrm{~m}$.

Запоминающий осциллограф с частотой дискретизации $f_{S}=2.5 \mathrm{GHz}$ фиксировал реализацию сигналаогибающей $e(t)$ для анализа распределений мгновенных значений его амплитуд. Эти распределения (рис. 2,a) являются бимодальными (двугорбыми). Левый „горб“ соответствует мгновенным значениям огибающей в отсутствие импульсов (межимпульсный интервал), правый - значениям огибающей принятого радиоимпульса. Представленные формы распределений сглажены методом сплайн-интерполяции. Распределения имеют „хвосты“, в которых сосредоточены как информативные, так и неинформативные мгновенные значения отсчетов реализации огибающей, вносящие ошибки при определении амплитуды импульсов.

Для устранения влияния неинформативных отсчетов были введены уровни отсечки $C_{L}$ и $C_{R}$ относительно высоты моды для каждого из горбов: при расчете амплитуды радиоимпульса использовались только те отсчеты, число которых превышало $C_{L}$ для левого горба и $C_{R}$ для правого горба. 

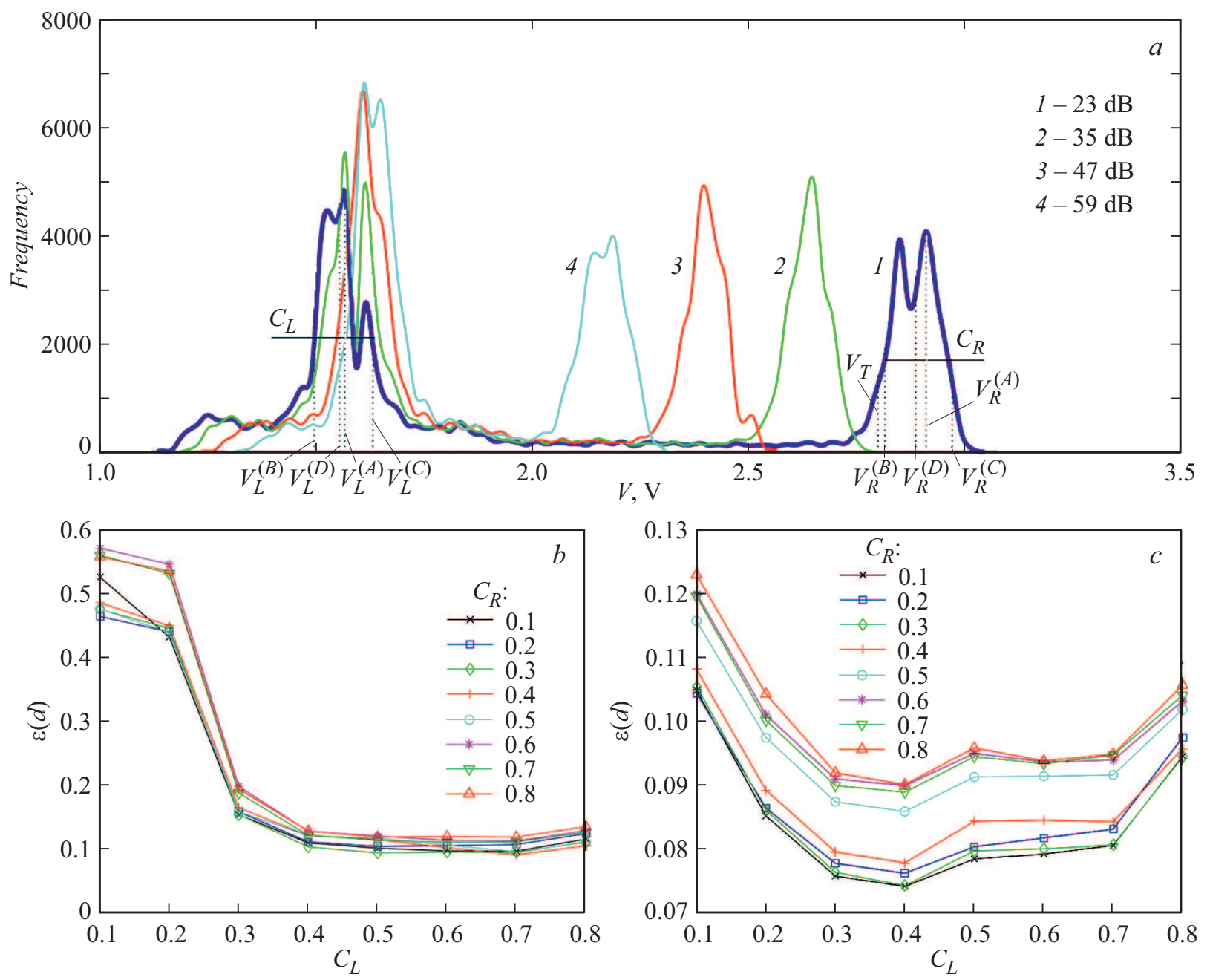

Рис. 2. Распределение мгновенных значений амплитуд сигнала-огибающей в приемнике, измеренное в эксперименте при различных значениях затухания между излучателем и приемником $(a)$, и оценки средней относительной ошибки измерения эквивалентного расстояния как функции относительных значений $C_{L}$ и $C_{R}$ уровней отсечки при использовании для оценки амплитуды правила $B(b)$ и правила $D(c)$.

Значение амплитуды $A_{d}$ огибающей радиоимпульса для заданного ослабления определялось как разность оценки напряжения $V_{L}$ для межимпульсного интервала и оценки напряжения $V_{R}$ огибающей принятого радиоимпульса при помощи следующих эвристических правил:

$A-$ разность между правой $V_{R}^{(A)}$ и левой $V_{L}^{(A)}$ модами горбов распределения (разность между локальными максимумами правого и левого горбов): $A_{d}^{(A)}=V_{R}^{(A)}-V_{L}^{(A)}$;

$B$ - разность между значениями амплитуды огибающей по уровню отсечки слева для обоих горбов распределения: $A_{d}^{(B)}=V_{R}^{(B)}-V_{L}^{(B)}$;

$C$ - разность между значениями амплитуды огибающей по уровню отсечки справа для обоих горбов распределения: $A_{d}^{(C)}=V_{R}^{(C)}-V_{L}^{(C)}$;

$D$ - разность между средними значениями амплитуды огибающей (среднее арифметическое значений отсчетов, лежащих выше уровня отсечки), для обоих горбов распределения: $A_{d}^{(D)}=V_{R}^{(D)}-V_{L}^{(D)}$.
Критерием точности служило значение средней относительной ошибки определения расстояния

$$
\varepsilon(d)=\frac{1}{m} \sum_{i=1}^{m} \frac{\left|\delta d_{i}\right|}{d_{i}},
$$

где $\delta d_{i}$ - ошибка измерения эквивалентного расстояния $d_{i}$, соответствующего ослаблению $Q_{i} ; m-$ число измерений.

Результаты вычисления средней относительной ошибки (3), полученные при помощи правил $B$ и $D$, в соответствии с (1) приведены на рис. 2, $b, c$. Ошибка представлена как функция относительных значений $C_{L}$ и $C_{R}$ уровня отсечки.

С ростом $C_{L}$ величина ошибки падает, достигая минимума в области $C_{L} \approx 0.3-0.6$ и $C_{R} \approx 0.1-0.4$, что объясняется отбрасыванием мгновенных значений амплитуд, составляющих хвосты распределений (рис. 2,a). Для дальнейших расчетов в качестве значений уровней отсечки выбраны значения $C_{L}=0.4$ и $C_{R}=0.3$ соответственно. 
Относительная ошибка определения расстояния для различных способов измерения амплитуды импульса $\left(C_{L}=0.4, C_{R}=0.3\right)$

\begin{tabular}{c|c|c|c|c|c|c}
\hline \multirow{2}{*}{$\begin{array}{c}\text { Относительная } \\
\text { ошибка } \varepsilon\end{array}$} & \multicolumn{6}{|c}{ Правило } \\
\cline { 2 - 7 } & $A$ & $B$ & $C$ & $D$ & BER & BER_C \\
\hline до $89 \mathrm{~m}$ & 0.176 & 0.092 & 0.069 & 0.074 & 0.206 & 0.090 \\
до $16 \mathrm{~m}$ & 0.170 & 0.054 & 0.042 & 0.044 & 0.124 & 0.053
\end{tabular}

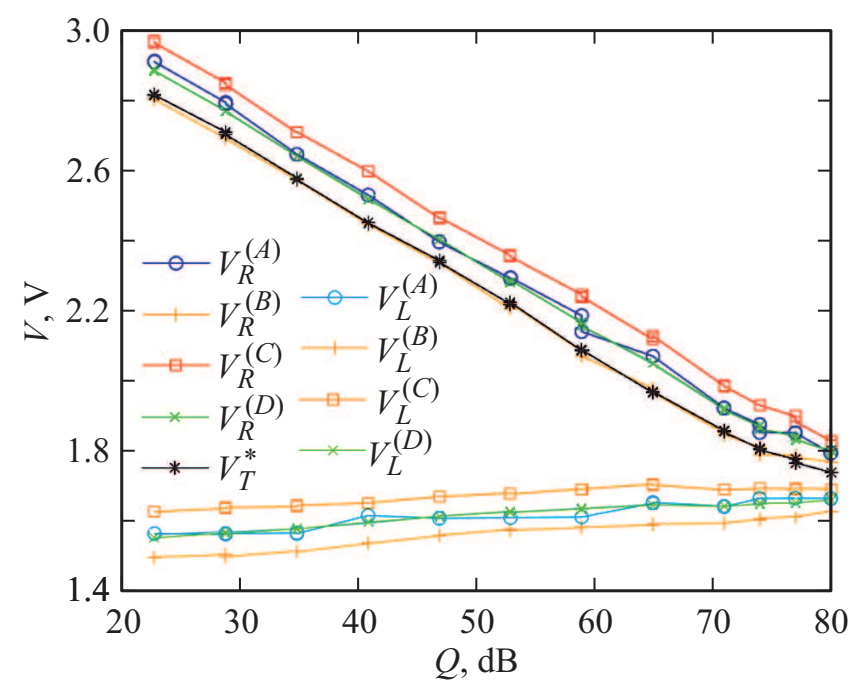

Рис. 3. Зависимости $V_{R}$ и $V_{L}$ принятого импульса от затухания $Q$ для правил $A, B, C, D$.

Результаты вычислений ошибки (3) приведены в таблице. Ошибка, получаемая при измерении амплитуды по правилу $A$ (по модам распределений), постоянна и равна 0.17. При использовании правил $B, C, D$ наилучший результат достигается при определении амплитуды по правому краю $(C)$ и среднему значению $(D)$ распределений, для которых ошибка (3) равна 0.07 в диапазоне изменения мощности $80 \mathrm{~dB}$ и 0.04 в диапазоне изменения мощности $65 \mathrm{~dB}$ (эквивалентное расстояние до $16 \mathrm{~m})$.

Вместе с тем проведенные измерения ошибки (3) по методике определения доли обнаруженных и пропущенных импульсов (2) показали, что для $F=0.9$ полученные значения $V_{T}^{*}$ дают относительную ошибку $\varepsilon(d)=0.2$ (см. таблицу, столбец BER). Поэтому практически вопрос заключается в том, каким образом приблизиться к точности, которую дает определение мощности сигнала по распределению его мгновенных амплитуд.

Из графика распределений мгновенных значений напряжения в приемнике (рис. 2,a) видно, что уровень напряжения, соответствующий межимпульсному интервалу, меняется в зависимости от мощности принятого сигнала. На рис. 3 приведена зависимость оценки значений $V_{R}$, полученной по правилам $A, B, C, D$ и по методу подсчета ошибок $\left.\left(V_{R}^{(A)}\right), V_{R}^{(B)}, V_{R}^{(C)}, V_{R}^{(D)}, V_{T}^{*}\right)$, и значений
$V_{L}\left(V_{L}^{(A)}, V_{L}^{(B)}, V_{L}^{(C)}, V_{L}^{(D)}\right)$ от затухания $Q_{i}$. Обнаружилось, что изменения этих величин согласованы между собой и зависят от затухания: при увеличении затухания сигнала значения $V_{R}$ монотонно убывают, а значения $V_{L}$ монотонно возрастают, что позволяет аппроксимировать эти значения линейными функциями $V_{R}=Q \operatorname{tg} \alpha+V_{R 0}$, $V_{L}=Q \operatorname{tg} \beta+V_{L 0}$, где $V_{R 0}, V_{L 0}$ - точки пересечения прямых с осью ординат, $\alpha, \beta-$ углы наклона прямых. Исключая $Q$, получим формулу для оценки напряжения $V_{L}$, соответствующего межимпульсному интервалу:

$$
V_{L}=\left(V_{R}-V_{R 0}\right) \frac{\operatorname{tg} \beta}{\operatorname{tg} \alpha}+V_{L 0} .
$$

Формулу (4) необходимо использовать для корректировки значения амплитуды $A_{d}$ принятых радиоимпульсов, измеренного методом подсчета ошибок обнаружения (2): по измеренному значению $V_{T}^{*}$, полученному при помощи определения доли обнаруженных и пропущенных импульсов, вычисляется по формуле (4) значение $V_{L}$, амплитуда принятых радиоимпульсов $A_{d}=V_{T}^{*}-V_{L}$ и делается оценка расстояния по формуле (1). В результате применения такого подхода ошибка (3) измерения расстояния снижается приблизительно в 2 раза (до 0.09 , столбец BER_C в таблице).

B работе показано, что относительная ошибка измерения расстояния составляет 9 и 5\% в диапазоне изменения мощности принимаемого сигнала до $80 \mathrm{~dB}$ (эквивалентное расстояние $89 \mathrm{~m}$ ) и до $65 \mathrm{~dB}(16 \mathrm{~m})$ соответственно. Полученные значения точности измерения расстояния позволяют использовать метод для практических приложений в существующей аппаратуре.

\section{Финансирование работы}

Работа выполнена в рамках государственного задания Института радиотехники и электроники им. В.А. Котельникова РАН.

\section{Конфликт интересов}

Авторы заявляют, что у них нет конфликта интересов.

\section{Список литературы}

[1] F. Zafari, A. Gkelias, K.K. Leung, IEEE Commun. Surv. Tutorials, 21 (3), 2568 (2019). DOI: 10.1109/COMST.2019.2911558

[2] A. Alarifi, A. Al-Salman, M. Alsaleh, A. Alnafessah, S. AlHadhrami, M.A. Al-Ammar, H.S. Al-Khalifa, Sensors, 16 (5), 707 (2016). DOI: 10.3390/s16050707

[3] A. Li, J. Fu, A. Yang, H. Shen, IEEE Access, 7, 13945 (2019). DOI: ACCESS.2019.2893398

[4] S. Subedi, H. Gang, N.Y. Ko, S. Hwang, J. Pyun, IEEE Access, 7, 31738 (2019). DOI: 10.1109/ACCESS.2019.2902564

[5] Y. Cheng, Y. Lin, IEEE Trans. Consum. Electron., 55 (3), 1295 (2009). DOI: 10.1109/TCE.2009.5277991 
[6] H. Lohrasbipeydeh, T.A. Gulliver, H. Amindavar, IEEE Trans. Signal Process., 62 (17), 4516 (2014).

DOI: $10.1109 /$ TSP.2014.2336634

[7] W. Li, Y. Jia, IET Signal Process., 9 (9), 681 (2015). DOI: 10.1049/iet-spr.2014.0477

[8] R.M. Vaghefi, M.R. Gholami, R.M. Buehrer, E.G. Strom, IEEE Trans. Signal Process., 61 (6), 1389 (2013). DOI: $10.1109 /$ TSP.2012.2232664

[9] C. Liang, F. Wen, IEEE Sensors J., 16 (5), 1265 (2016). DOI: 10.1109/JSEN.2015.2500270

[10] J. Zhu, Q. Chen, J. Zhang, in 2017 IEEE 9th Int. Conf. on communication software and networks (ICCSN) (Guangzhou, China, 2017), p. 830. DOI: 10.1109/ICCSN.2017.8230228

[11] G.M. Mendoza-Silva, J. Torres-Sospedra, J. Huerta, Sensors, 19 (20), 4507 (2019). https://doi.org/10.3390/s19204507

[12] Yu.V. Andreyev, A.S. Dmitriev, E.V. Efremova, A.D. Khilinsky, L.V. Kuzmin, Int. J. Bifurc. Chaos, 15 (11), 3639 (2005). DOI: $10.1142 / \mathrm{S} 0218127405014295$

[13] Е.В. Ефремова, А.С. Дмитриев, Л.В. Кузьмин, Письма в ЖТФ, 45 (17), 3 (2019).

DOI: 10.21883/PJTF.2019.17.48214.17865 [Пер. версия: 10.1134/S1063785019090037].

[14] A.S. Dmitriev, L.V. Kuzmin, A.I. Ryshov, Y.V. Andreyev, M.G. Popov, in 2018 Engineering and Telecommunication (EnT-MIPT) (M., 2018), p. 76. DOI: 10.1109/EnT-MIPT.2018.00023 